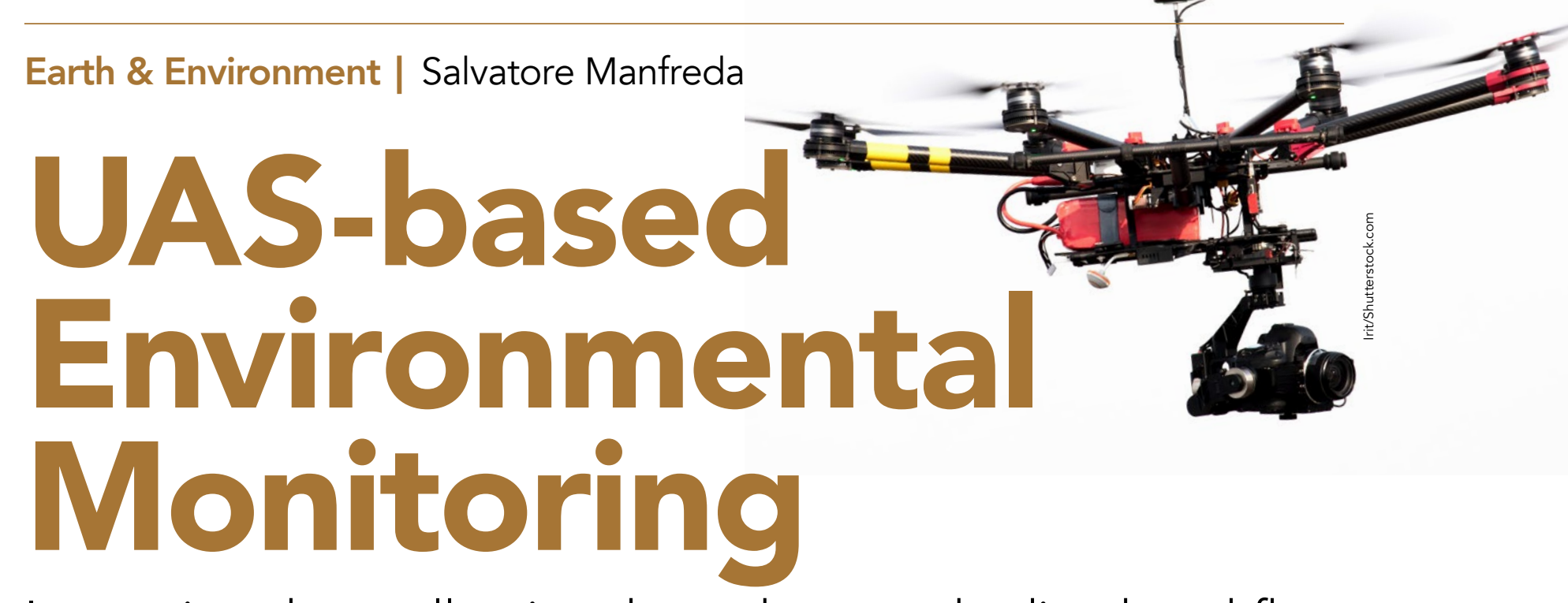

Improving data collection through a standardised workflow

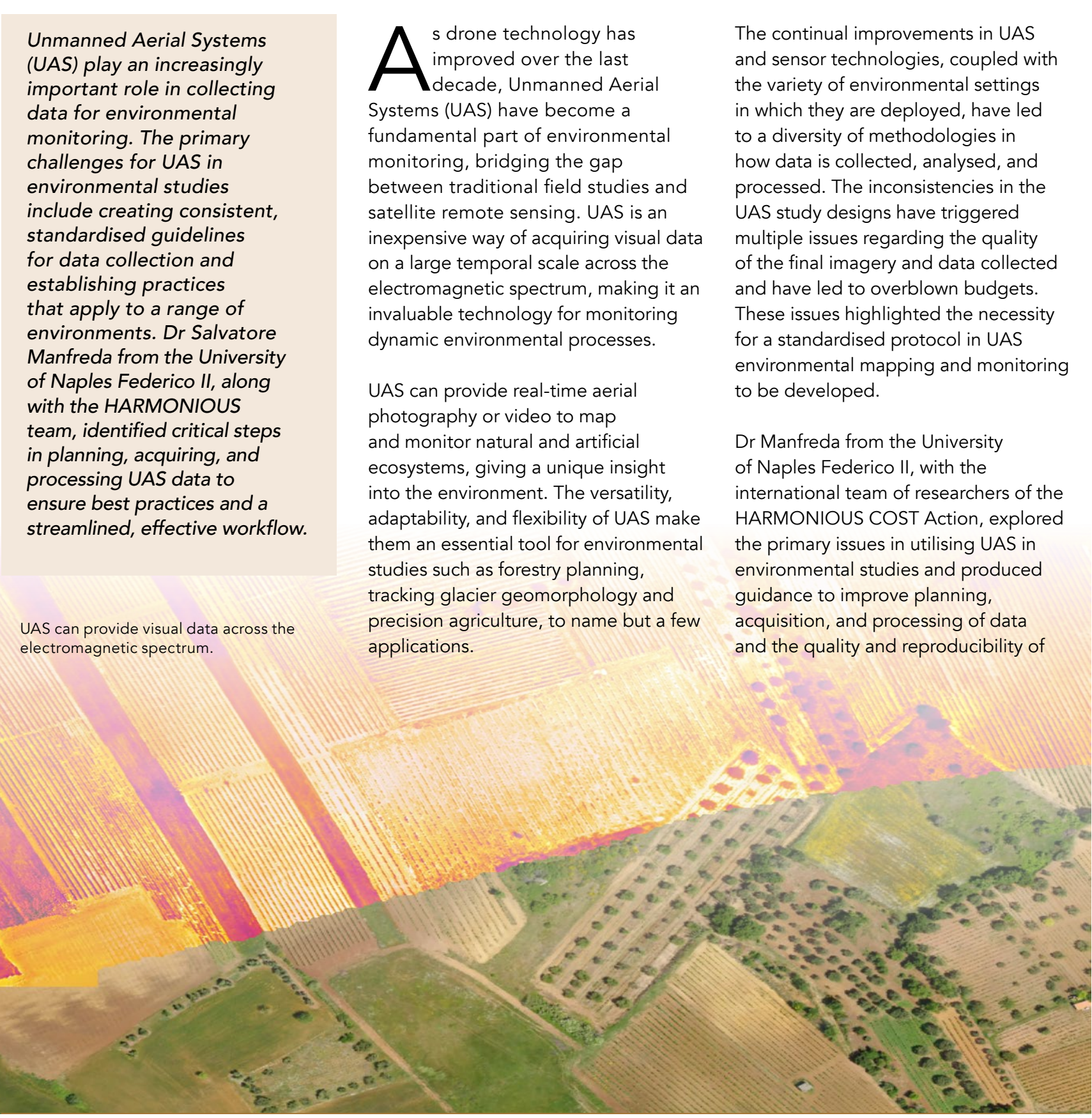

Unmanned Aerial Systems UAS) play an increasingly monitoring. The primary workflow methodology with five 2) pre-flight fieldwork; 3) flight mission; 4) processing of aerial data; and 5) quality assurance.

\section{UAS LIMITATIONS}

ic, temporal, and qualitative benefits in using UAS over satellites or manned aircraft, whic are limited by their cost and how often a survey can use them. However, as UAS is still an immature technology, limitations exist in how data is collected and analysed.

Previous studies have indicated that many UAS surveys fail to consider the planning and processing of UAS imagery Whon the speed and height sensors are not considered in the planning stage, and the weather is not accounted for on the day of the flight the UAS imagery will be blurred or of incorrect resolution.

These limitations could be mitigated through a structure of standardisation which can work as a checklist for UAS surveys to ensure accurate collection

STANDARDISING UAS DATA

\section{COLLECTION}

Although every UAS survey will be slightly different owing to the wide variety of vegetation, topography, study environments, a stan ir workflow, which accounts for every stage of the survey and applies to every environment, will be incredibly beneficial in assuring appropriate planning for high-quality results.

Through creating a generalised workflow in five interrelated steps, HARMONIOUS's research aims to improve the final quality of data and analysis. The workflow was designe based on harmonising multiple methods collated from recent research and reviews of different UAS surveys.

\section{WORKFLOW DESIGN}

Every UAS study can vary greatly and therefore requires a bespoke study
design to set out a detailed mission

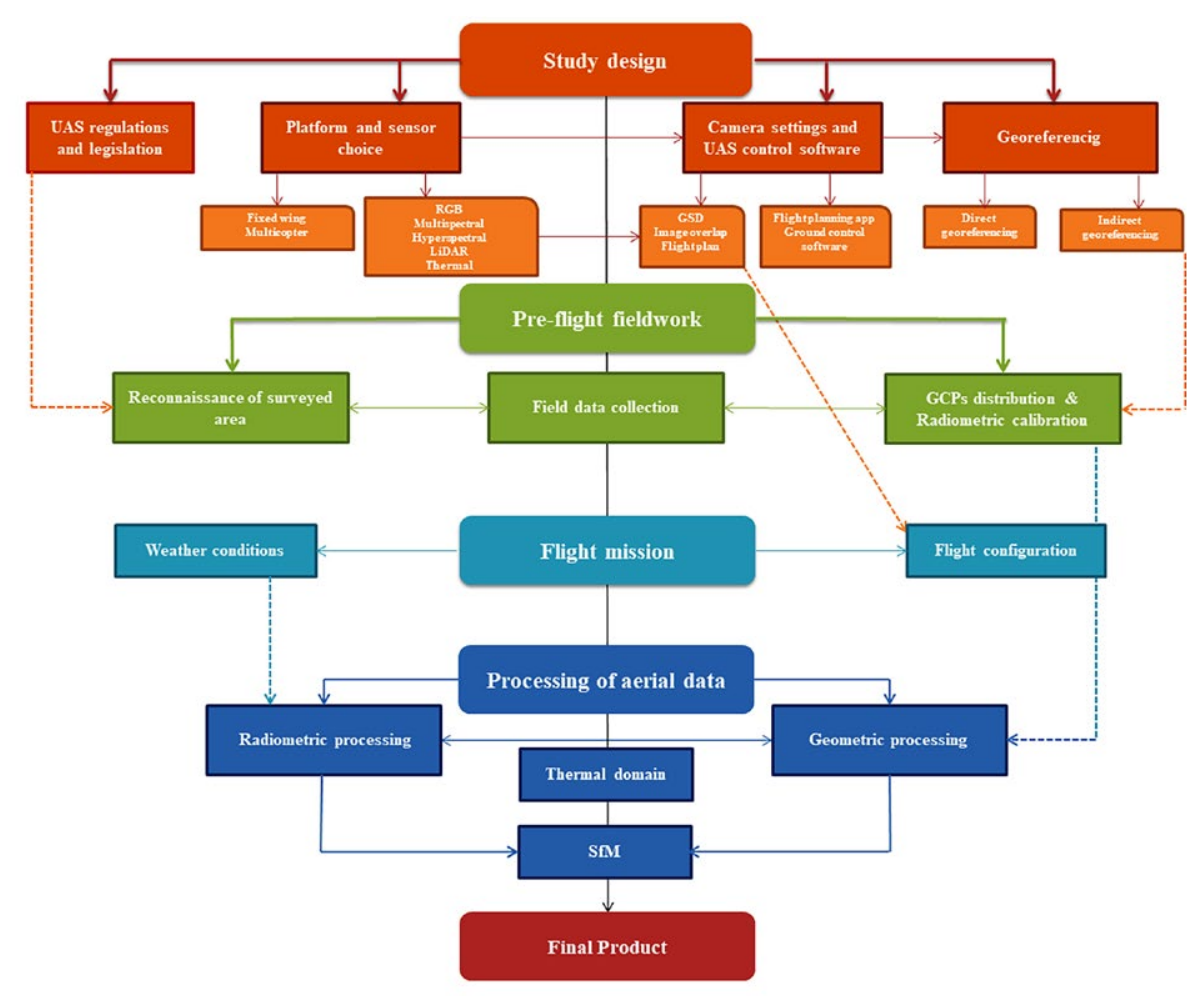

Workflow: Each mission requires a bespoke study design. Then, a pre-flight study takes place before
the flight mission. The final stage describes how to best process the imagery and data from the flight.

plan for the study area. Consequently, to ensure the mission will get the initial step in the workflow process permission to fly in the study area. is to design the study; this step is The platform, sensor, camera essential to set up the parameters of settings, and UAS control software the survey and consider the specifics choices are purely dependent of the environment and the research on the survey's requirements and question as this will shape where, how, limitations - concerning budget and and when the flight can take place and time limitations, or the image quality, what sensors will be used. spectral and spatial resolution, and the When all factors are considered, the survey area's size. Finally, in the study study design can be an incredibly be conducted to ensure the imagery is

There are clear economic, temporal and qualitative benefits in using UAS over satellites or manned aircraft.

complex problem. The final quality of the model is dependent on all of these accounted for

In general, mission plans for environmental studies focus on four primary elements: 1) UAS regulations and legislation, 2) plattorm and sensor choice 3) camera seltings and UAS control software and 4) target geo-referencing.

Local UAS regulations and legislation will have to be understood first taken correctly. The best way to do this is to find ground control points (GCP) for reference.

Once the study design is complete, the next step in the workflow is to conduct a pre-flight study. This section of the workflow entails reconnaissance and a terrestrial reconnaissance will reveal take-off and the flight to be georefernced. The survey of the survey area. The area's landing points, any possible visual or 


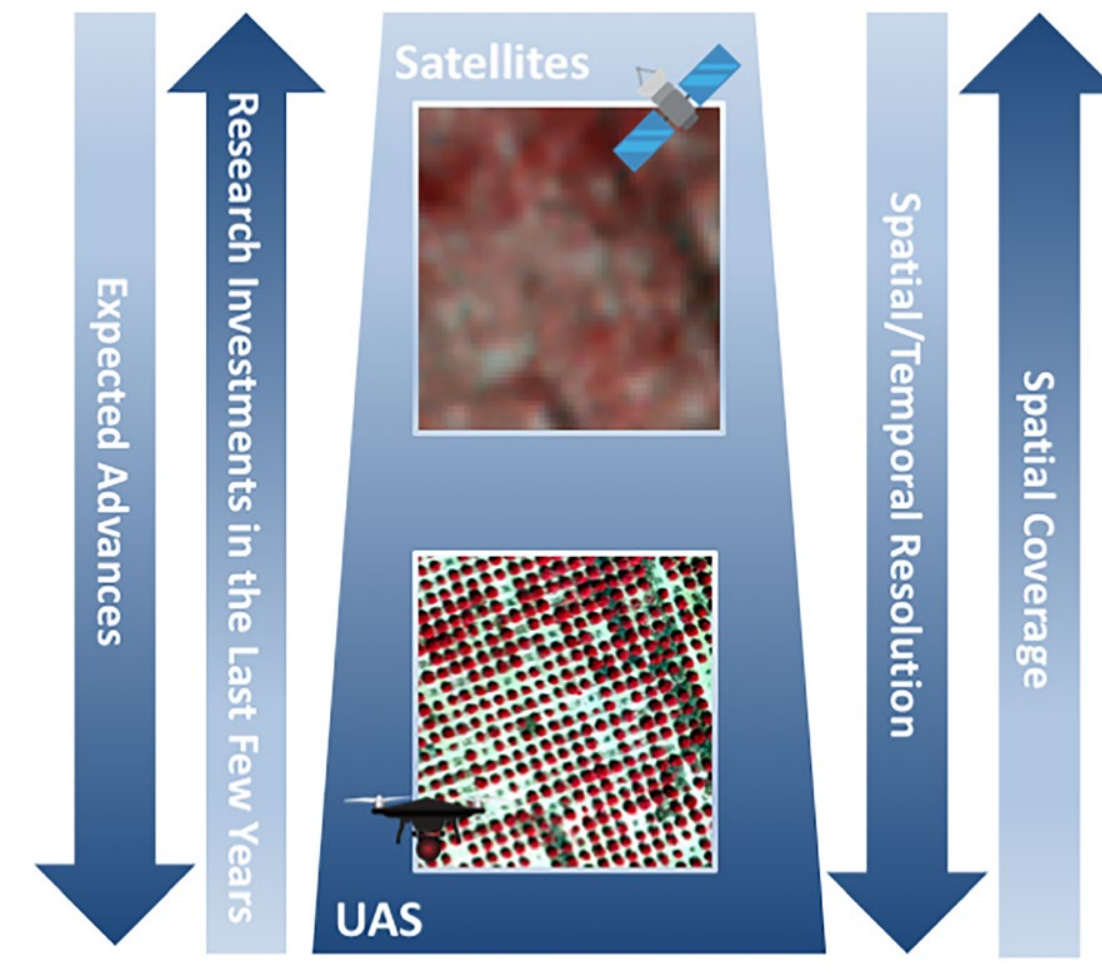

There are clear economic, temporal, and qualitative benefits in using UAS over satellites. However
UAS is still an immature technology and limitations exist in how data is collected and anlysed.

The researchers have created a harmonised workflow that will be an essential element of any UAS survey in the future.

field study will be highly dependent Following the pre-flight, the workflow on the environmental medium being explains how to safely and most studied but will supplement and the effectively conduct the flight itself. The
influence any data collected from the challenge at this stage is to account for influence any data collected from the challenge at this stage is to account for
UAS study.

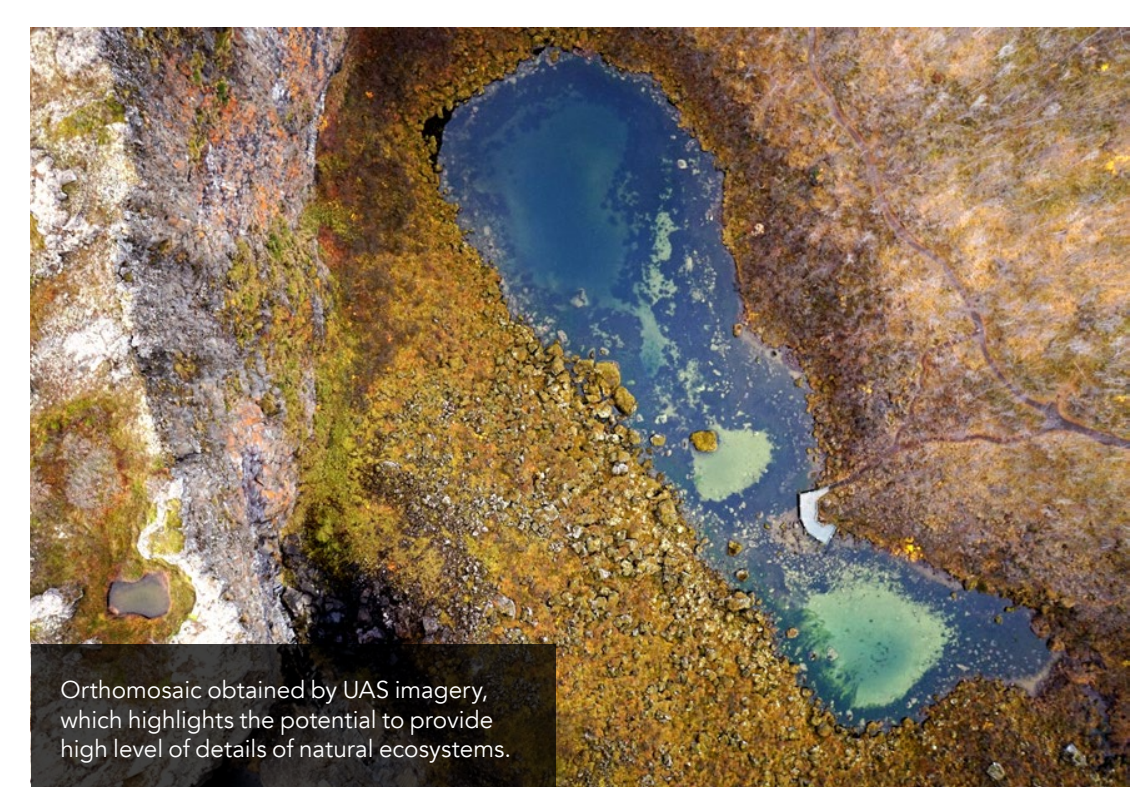

humidity, light levels, and fog can affect data quality, so it must be compensa

The final stage in the workflow describes how to best process the imagery and data from the flight. When processing, it is essential for the surveyors to account for the distortions, often in UAS imagery. These can misrepresent the radiometrics and geometrics of the study object. However, a series of steps quantify the radiometric or geometric problems, for which there is a corrective method.

A critical aspect of HARMONIOUS's method is that quality assurance must

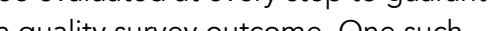
way alluded to, which can save time and money and ensure quality images, uses a portable resolution test chart. These charts, when used correctly, can give assurances that cameras are calibrated correctly before the flight takes place.

\section{A NEW STANDARD PRACTICE} Recent advances in UAS have mean that low-cost and near real-time data collection has become possible in an array of environmental studies. With their essential work, Dr Manfreda and his fellow researchers have created a harmonised workflow and accompanying checklists that will be a vitele fure, fur he thy the efficacy orUAS in studying the environment.

The researchers have designed the workflow to reduce error in data collection and processing and ensure flights are conducted within budget, safely, and effectively. This research will undoubtedly improve future UAS studies and be a template by which all reviews can be guided, streamlining the study process and making results easily reproducible.

HARMONIOUS's research assists in furthering UAS procedures an ensuring that UAS studies in the future will have more accurate results referenced in this article.

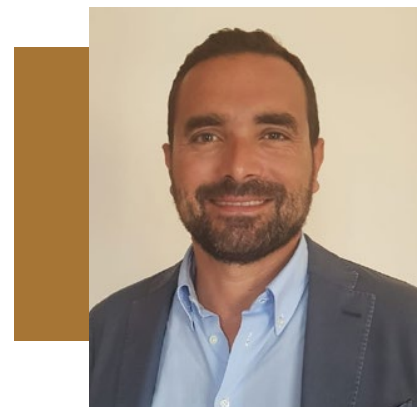

\section{Behind the Research}

\section{Dr Salvatore Manfreda}

E: salvatore.manfreda@unina.it T: $+390971205140 \quad$ W: https://www.salvatoremanfreda.it/

Research Objectives

COST Action HARMONIOUS, coordinated by Prof anfreda, provides practical guidelines for the use of

\section{Detail}

Salvatore Manfreda

Dipartimento di Ingegneria Civile, Edile e Ambientale (1)

via Claudio, 21, 80125 Napoli, Italy

Bio

Salvatore Manfreda is full Professor of Water Management, Hydrology and Hydraulic Constructions at the University of Naples Federico II. He is currently the Chair of the COST Action HARMONIOUSHarmonization of UAS techniques for agricultural and natural ecosystems monitoring. His research activities focus on distributed modelling, flood risk, stochastic processes in hydrology and UAS-based monitoring.

\section{Funding}

CA research was funded by the COST Action CAI6219 HARMONIOUS-Harmonization of ecosystems monitoring".

\section{Collaborators}

Brigitta Toth, Sorin Herban, Corine Davis, Antonino Maltese, Felix Frances, Jana Müllerová, Yijian Zeng, Anna Brook, Matthew Perks, Dariia Strelnikova, Eyal Ben-Dor, Flavia Tauro, László Bertalan, Bob Su, Petr Dvořák, Sander Mücher, Giuseppe Ciraolo. Yijian Zeng, Zhongbo Su, Richard Lucas, Nunzio Romano, David Helman, Lindon Estes, Kelly Caylor, M. Kohv, Guiomar Ruiz-Pérez, Giulia Vico, Xurxo Gago, Ruodan Zhuang, Goran Tmusic, Helge Aasen Mike James, Gil Concalves, Maria Polinova, Jose Juan Arranz, Janos Meszaros, Kasper Johansen, Yoann Malbeteau, Matthew McCabe, Isabel Pedroso de Lima, Sophie Pearce, Robert Ljubicic Salvador Peña-Haro, Alonso Pizarro, Silvan Sasso, Salvatore Grimaldi, lan Maddock, , Adrict João L. M. P. de Lima

\section{References}

Manfreda, S., McCabe, M.F. et al. (2018). On the Use of Unmanned Aerial Systems for Environmental Monitoring. Remote Sensing, 10(4), 641. Available at: https://doi. org/10.3390/rs1004064

Tmušić, G., Manfreda, S., et al. (2020). Current Practice in UAS-based Environmental Surveying. Remote Sensing rs12061001

\section{Personal Response}

As new iterations of UAS technologies are developed, could the workflow process become more automated?

II We are now focusing on the preparation of a book for UAS applications in environmental monitoring.
for

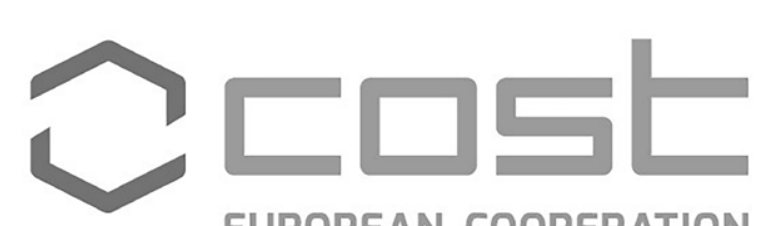
EUROPEAN COOPERATION IN SCIENCE \& TECHNOLOGY

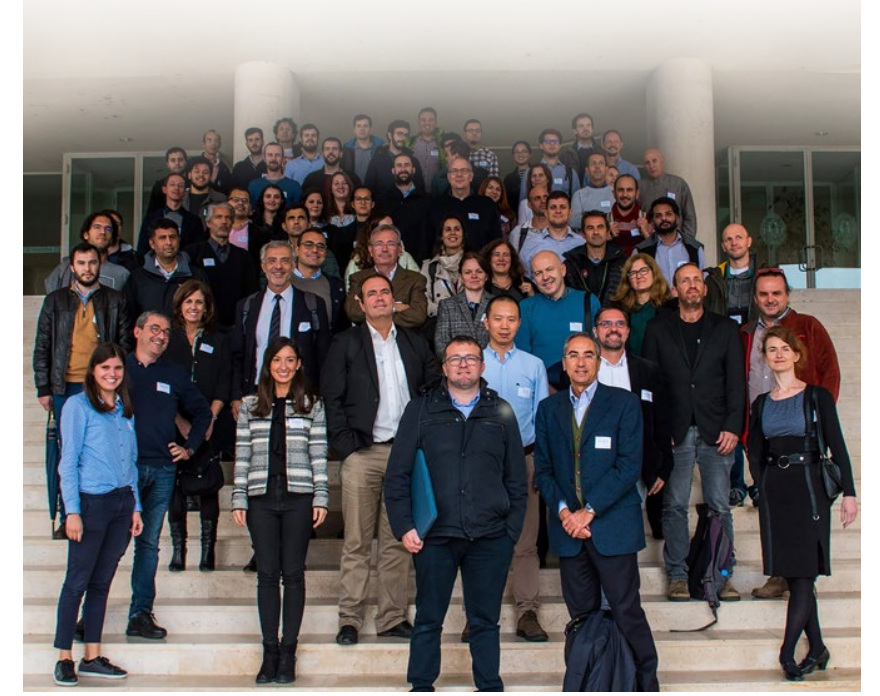

